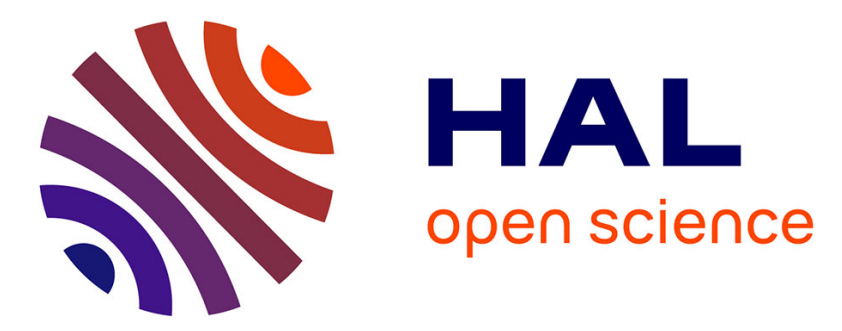

\title{
Un état francophone du champ du management des connaissances : la communauté GeCSO
}

Jean-Louis Ermine, Pascal Lièvre, Claude Paraponaris, Claude Guittard

\section{To cite this version:}

Jean-Louis Ermine, Pascal Lièvre, Claude Paraponaris, Claude Guittard. Un état francophone du champ du management des connaissances: la communauté GeCSO. Revue management \& avenir, 2014, 67, pp.56-77. 10.3917/mav.067.0056 . hal-01025287

\section{HAL Id: hal-01025287 \\ https://hal.science/hal-01025287}

Submitted on 4 Dec 2014

HAL is a multi-disciplinary open access archive for the deposit and dissemination of scientific research documents, whether they are published or not. The documents may come from teaching and research institutions in France or abroad, or from public or private research centers.
L'archive ouverte pluridisciplinaire $\mathbf{H A L}$, est destinée au dépôt et à la diffusion de documents scientifiques de niveau recherche, publiés ou non, émanant des établissements d'enseignement et de recherche français ou étrangers, des laboratoires publics ou privés. 


\title{
UN ÉTAT FRANCOPHONE DU CHAMP DU MANAGEMENT DES CONNAISSANCES : LA COMMUNAUTÉ GECSO
}

\author{
Jean-Louis Ermine et al.
}

Management Prospective Ed. | Management \& Avenir

\author{
$2014 / 1-N^{\circ} 67$ \\ pages 56 à 77
}

ISSN 1768-5958

Article disponible en ligne à l'adresse:

http://www.cairn.info/revue-management-et-avenir-2014-1-page-56.htm

Pour citer cet article :

Ermine Jean-Louis et al., « Un état francophone du champ du management des connaissances : la communauté GeCSO »,

Management \& Avenir, 2014/1 №67, p. 56-77. DOI : 10.3917/mav.067.0056

Distribution électronique Cairn.info pour Management Prospective Ed.

(c) Management Prospective Ed.. Tous droits réservés pour tous pays.

La reproduction ou représentation de cet article, notamment par photocopie, n'est autorisée que dans les limites des conditions générales d'utilisation du site ou, le cas échéant, des conditions générales de la licence souscrite par votre établissement. Toute autre reproduction ou représentation, en tout ou partie, sous quelque forme et de quelque manière que ce soit, est interdite sauf accord préalable et écrit de l'éditeur, en dehors des cas prévus par la législation en vigueur en France. II est précisé que son stockage dans une base de données est également interdit. 


\title{
Un état francophone du champ du management des connaissances : la communauté GeCSO
}

\author{
par Jean-Louis Ermine ${ }^{42}$, Pascal Lièvre ${ }^{43}$, \\ Claude Paraponaris ${ }^{44}$ et Claude Guittard ${ }^{45}$
}

Résumé

L'émergence d'une communauté de recherche francophone multidisciplinaire GecSO (Gestion des Connaissances, Société et Organisation) autour du management des connaissances donne lieu à la production d'une centaine de contributions depuis 2008. L'objet de cet article est de proposer une structuration du champ à partir de cette production. II est possible de dégager six programmes de recherche qui sont à la fois distincts mais reliés entre eux. Ces programmes constituent des attracteurs propres à structurer le champ. Ces programmes de recherche ont la particularité de traverser largement les disciplines. Ces programmes de recherche convergent sur la nécessité de distinguer information et connaissance, d'appréhender la connaissance comme une activité cognitive plutôt qu'un objet.

\section{Abstract}

The emergence of a French speaking research community in Knowledge Management (through the annual GeCSO congress), produced more than one hundred contributions since 2008. This article proposes a structure for that domain, based on that production. It is possible to extract six research programs that are distinct but tightly linked. Those programs are attractors liable to structure the domain. Those research programs are widely multidisciplinary. They converge on the necessity of making a distinction between information and knowledge, and considering knowledge more as a cognitive process than an object.

L'émergence d'une communauté de recherche francophone multidisciplinaire GeCSO (Gestion des Connaissances, Société et Organisation) autour du management des connaissances a donné lieu à la production d'une centaine de contributions depuis 2008 dans le cadre d'un colloque organisé chaque année. L’idée de proposer un état du champ du management des connaissances à partir

\footnotetext{
42. Jean-Louis Ermine, Institut Telecom, Telecom Business School, jean-louis.ermine@it-sudparis.eu

43. Pascal LiėVre, Maître de Conférences HDR, Université d'Auvergne, CRCGM EA 3849, pascallievre@orange.fr

44. Claude Paraponaris, Professeur des universités, Université Aix-Marseille, LEST CNRS UMR 7123,

claude.paraponaris@univ-amu.fr

45. Claude GuittaRd, BETA, Université de Strasbourg, guittard@unistra.fr
} 


\section{Un état francophone du champ du management des connaissances : la communauté GeCSO}

de ce matériaux s'est fait jour au sein de cette communauté ${ }^{46}$. Une première structuration du champ du management des connaissances a été proposée à partir des sept thèmes recensés comme attracteurs et hiérarchisés par ordre d'importance dans l'ensemble des contributions (Paraponaris, Ermine, Guittard, Lièvre, 2012) à savoir la génération des connaissances à partir des activités, les fondements épistémologiques du savoir comme action située, le transfert de connaissance, la capitalisation des connaissances, la modélisation des connaissances, les communautés épistémiques et les communautés de pratiques, l'innovation et connaissance. Nous structurons le champ en proposant un regroupement ordonné de ces différents thèmes, en leur donnant la structure de de programmes de recherche articulés entre eux et prenant la forme d'une matrice composée de trois lignes et trois colonnes. Cette matrice permet de rendre compte à la fois de la spécificité de chaque programme mais aussi des relations entre ces programmes. Trois programmes de recherche structurent profondément le champ sur le plan historique, nous les avons positionnés en ligne, les trois autres programmes sont des entrées sur des opérations classiques en matière de management des connaissances, nous les avons placés en colonne. II faut considérer ces programmes comme des manières différentes de rentrer dans le champ et d'y circuler. Nous illustrons par quelques contributions GecSO les différentes entrées de cette matrice.

Nous commençons par les lignes. Trois programmes de recherche peuvent être distingués dont les origines sont très différentes. Un premier programme est centré autour des travaux de Jean-Louis Ermine $(1996,2003,2008)$ et du modèle MASK dont le point de départ est une organisation à risque comme le CEA (Commissariat à l'Energie Atomique) qui se pose la question de la formalisation du retour d'expérience. Aucune organisation à risque ne peut fonctionner comme le rappelait Georges Yves Kerven (1996) sans se nourrir de cette connaissance particulière qu'est le retour d'expérience. Jean-Louis Ermine construit une méthode formelle de modélisation des connaissances, à forte capacité intégrative sur le plan théorique. Le point de départ de ce modèle est l'articulation entre la théorie du système générale de Le Moigne (1994) et le modèle SECI de Nonaka et Takeuchi (1995). Nous intitulons ce programme : des organisations à risque à la modélisation des connaissances.

Un second programme est centré autour des travaux de Patrick Cohendet sur les communautés de savoir (Amin, Cohendet, 2003 ; Cohendet, Crepet, Dupouet, 2005). Le point de départ est complètement différent. C'est le constat de l'émergence d'une nouvelle forme de configuration de l'économie

\footnotetext{
46. Claude Paraponaris a bien voulu tenter l'aventure et a proposé une première mise en perspective lors d'une intervention d'ouverture à Clermont-Ferrand en 2011. Un collectif s'est constitué autour de ce projet rassemblant Jean-Louis Ermine, l'initiateur de cette communauté, Claude Guittard, l'artisan du colloque de 2010 au Beta à Strasbourg, et Pascal Lièvre, qui avait pris la responsabilité du colloque de 2011. Une première version sous la direction de Claude Paraponaris a vu le jour dans le cadre d'un article pour la revue VINE : The Journal Of Information and Knowledge Management : "Knowledge Management in a French Research Community - A Case Study of GeCSO Congress ». Pascal Lièvre a pris le relais pour engager à partir des premiers matériaux mis en évidence une deuxième mise en perspective dans le cadre de ce cahier spécial de la revue Management \& Avenir.
} 


\section{7 \\ management \\ Qr avenir}

contemporaine, une économie de l'innovation fondée sur la connaissance qui bouleverse profondément les règles du jeu managérial. La connaissance devient le fondement de la création de la valeur de l'entreprise parce qu'elle permet l'innovation. Or, il apparaît que ce sont les communautés en tant que forme social spécifique qui permettent l'émergence, la circulation et la capitalisation des connaissances. Ces communautés apparaissent fondamentalement comme informelles et auto-organisées. Quel est le véritable statut "social » de ces communautés ? Comment peut-on les piloter? Nous dénommons ce programme de l'économie de la connaissance aux pilotages des communautés de savoir.

Le troisième programme de recherche est plus éclaté. II s'intéresse à la mutation paradigmatique du champ du management des connaissances telle que l'exprime par exemple un ouvrage collectif dirigé par Mclnerney and Day : Rethinking Knowledge Management (2007) où la connaissance n'est plus appréhendée comme un objet mais plutôt comme un processus cognitif. Ce programme de recherche se pose d'emblée des questions épistémologiques et interpelle les pratiques du management des connaissances dans les organisations à partir de cette mutation paradigmatique. On retrouve dans ce programme qui n'est pas unifié comme le sont les deux autres programmes que nous avons cité, un certain nombre de contributions comme celles de Lièvre et Lièvre-Rix (2010, 2011), de Hachour et Abouad (2008) et celles de Brassac (2008). Nous appelons ce programme les fondements épistémologiques du management des connaissances.

On peut aussi identifier trois autres programmes de recherche qui sont plus ou moins liés aux précédents, mais aussi reliés entre eux, en entrant par les colonnes qui recouvrent des opérations types associées au management des connaissances : la génération des connaissances, le transfert des connaissances et l'accumulation des connaissances. Dans la communauté GeCSO, le programme sur la génération des connaissances est plutôt centré sur la conception de projet et le cycle de vie du produit. Nous pouvons identifier les travaux de Belleval, Deniaud, Lerch (2010) sur un modèle de conception à base de réseau de contradiction, ceux de l'Ecole Bordelaise (Brunel, Girard, Moradi, Vallespir, Zolghadri, 2008, 2010) qui vise à modéliser un cycle dit d'ingénition en se proposant d'articuler trois corpus théorique : conception, connaissance et apprentissage. Un programme sur le transfert des connaissances qui prend différentes orientations : a) le processus même de transfert de connaissance sous l'angle de la psychologie sociale des processus cognitifs (Brassac, 2008), b) le transfert par le développement des compétences (Coulet, 2011) et c) le transfert des connaissances au moyen de dispositif de formation (Wannemacher, 2010 ; Gadille, Machado, 2011). Et enfin le dernier programme centré sur la capitalisation des connaissances a deux axes : le programme autour des retours d'expérience (REX ou RETEX) (Gautier, 2010 ; Godé et Barbaroux, 2011 ; 


\section{Un état francophone du champ du management des connaissances : la communauté GeCSO}

Rachedi, Boujut, Poussier, 2010) et la captation des gestes techniques à partir de dispositif vidéo (Le Bellu, Lahlou, Le Blanc, 2009).

Nous proposons d'exposer dans un premier temps les programmes de recherche à caractère général et dans un deuxième temps, les programmes de recherche centrés sur des opérations classiques de management des connaissances.

\section{Des programmes généraux de structuration du champ du management des connaissances}

\subsection{Des organisations à risque à la modélisation des connaissances : avec et autour de MASK}

De nombreux travaux font référence à la méthode MASK (Method for Analysing and Structuring Knowledge) développée par Jean-Louis Ermine (1996, 2008). Cette méthode, issue d'un processus constant de fertilisation entre études théoriques et expérimentations pratiques, se constitue depuis plus d'une quinzaine d'années et a désormais atteint une robustesse qui peut désormais permettre son déploiement à large échelle. Une centaine d'organisation dans des secteurs très variés ont utilisé cette méthode. La méthode MASK repose sur deux principes fondamentaux : toute organisation détient un savoir organisationnel en propre et la complexité de ce savoir nécessite une modélisation spécifique.

Le premier principe est qu'une entreprise (ou organisation en général) détient un « savoir organisationnel », qui lui est propre. Ce savoir est bien plus que la somme des savoirs individuels des employés, et il se pérennise plus ou moins à travers le temps, via des produits d'information (documents, bases de données, logiciels...) ou via des échanges/transferts individuels et/ou collectifs. II s'accumule dans l'entreprise au cours de son histoire, et forme ce qu'on appelle le "Patrimoine de Connaissances ". L'existence de ce patrimoine en tant que sous-système (intangible) de l'entreprise est encore controversée, car il correspond à une remise en cause de la vision classique qui assimile cette dernière à un système traitant de l'information pour des acteurs opérationnels ou décisionnaires. Cette nouvelle vision de l'organisation, comme " processeur de connaissances", peut être représentée par le schéma systémique, dit AlK, (Actors, Information, Knowledge), qui prend en compte les flux de connaissances qui circulent dans l'organisation (Ermine, 2008). Ce modèle d'organisation AlK, représente un système où des réseaux d'acteurs de la connaissance $(A)$ interagissent avec un système d'information (I). Ce système produit et consomme des connaissances. Ces connaissances s'accumulent dans le patrimoine de connaissances $(K)$ de l'organisation. 


\section{7 \\ management \\ Qr avenir}

Le deuxième principe est que la connaissance organisationnelle (le système $\mathrm{K}$ ) est un système complexe, elle n'est donc intelligible et maîtrisable (pour des fins de gestion) qu'à travers une représentation plurielle, sous plusieurs points de vue. Ces points de vue sont en nombre réduits, et suffisamment génériques pour s'appliquer à tous les systèmes de connaissances, quel que soit le domaine d'application. De plus, comme on l'a déjà dit, la partie tacite de cette connaissance est largement majoritaire. Basée sur ces deux principes, la méthode MASK, dans sa plus récente version, comprend quatre phases qui réalisent le « cercle vertueux du $\mathrm{KM}$ ».

Phase 1 : L'analyse stratégique du patrimoine de connaissances. Le patrimoine de connaissances d'une organisation est considéré de nos jours comme un de ses actifs les plus stratégiques. Comme nous venons de le voir, ce patrimoine est vulnérable et menacé par des pertes massives de connaissances (tacites pour la plupart). Un plan massif de préservation et de transfert doit donc être envisagé, et intégré comme un processus stratégique de l'organisation. Mais alors se posent un grand nombre de questions : quelles sont les connaissances vraiment menacées ? Sont-elles vraiment stratégiques pour l'organisation ? Qui est porteur de ces connaissances ? Quels sont les types d'actions opérationnelles à envisager ? Comment garantir que le plan d'action qui va être mis en place à moyen terme est en phase avec les objectifs stratégiques de l'organisation ? Etc. Pour tenter de répondre à ces questions, il est donc nécessaire d'effectuer un audit du patrimoine de connaissances guidé par la stratégie de l'organisation, et de proposer un plan d'action de préservation et de transfert qui est aligné avec cette stratégie. C'est cette première phase qui est appelée « analyse stratégique du patrimoine de connaissances », qui a pour but de repérer les domaines de connaissances qui sont « critiques » dans l'organisation.

Phase 2 : La capitalisation du patrimoine de connaissances. Parmi les connaissances critiques identifiées dans la première phase, un grand nombre relèvent d'une action de capitalisation. Cette phase concerne tout domaine de connaissances critique et stratégique, à forte composante tacite, où la partie tacite est essentiellement détenue par des experts identifiés. Dans ce cas, la capitalisation s'entend comme le recueil de connaissances auprès des experts, afin de formaliser leurs savoirs non-écrits, en vue de les faire partager à d'autres personnes du même métier, ou exerçant des activités très proches.

Phase 3 : Le transfert du patrimoine de connaissances. La capitalisation permet de recueillir et de structurer le contenu sensible d'un domaine de connaissance, et ainsi de constituer un corpus de connaissances (ou référentiel) du domaine. II s'agit ensuite de transférer, à partir de ce corpus, la connaissance à une communauté qui doit l'utiliser pour ses pratiques opérationnelles. C'est la véritable problématique du transfert qui se pose là : comment concevoir des dispositifs de transfert à partir du corpus de connaissances constitué, en fonction de l'objectif, la cible, l'environnement, etc. ? 


\section{Un état francophone du champ du management des connaissances : la communauté GeCSO}

Phase 4 : L'innovation fondée sur les connaissances. Pour atteindre le but final de toute organisation actuelle : être une organisation créatrice, le processus KM doit s'achever par la capacité de l'organisation à faire évoluer son patrimoine de connaissances dans une perspective stratégique. Ceci implique que toutes les ressources crées dans les phases précédentes doivent être utilisées comme un levier de l'innovation à travers la création de connaissances. Pour ces quatre phases, MASK utilise des outils tels que les cartes cognitives (cartes stratégiques, cartes des domaines de connaissance), des grilles d'analyses ("Critical Knowledge Factors »), des outils de modélisation cognitive (tâches, activités, concepts, lignées, phénomènes...), des modèles de transfert, des modèles d'évolution...

Au sein de la communauté GeCSO, des auteurs mobilisent et discutent la modélisation des connaissances proposée par MASK : Bonnet \& Lièvre, 2010 ; Brahami \& Atmani, 2009 ; Brunel, Zolghadri \& Girard, 2008 ; Fatallah, Stal-Le Cardinal, Ermine \& Bocquet, 2010 ; Moradi \& Vallespir, 2009 ; Ricciardi, Barroso \& Ermine, 2011 ; Saulais \& Ermine, 2011, 2012 ; Soulignac, Ermine, Paris, Devise \& Chanet, 2011.

\subsection{De l'économie de la connaissance aux pilotages des communautés de savoir}

Le point de départ de ce programme est le champ de l'économie. Des économistes dont Patrick Cohendet (Amin, Cohendet, 2004 ; Cohendet, Créplet, Dupouet, 2005) font état de l'émergence d'un nouveau régime de croissance de l'économie qui prend la forme d'une économie fondée sur la connaissance (OCDE, 2002). Cette nouvelle forme de l'économie se traduit par : a) la montée en puissance dans la production des capitaux intangibles (capital humain, social et intellectuel), b) l'apparition brutale des nouvelles technologies de l'information et de la communication et $\mathrm{c}$ ) l'innovation comme nouvelle règle du jeu concurrentiel. Les économistes investissent ce qui fonde la valeur de cette nouvelle économie qui est un objet d'une grande complexité : la connaissance. II apparaît que les communautés informelles et auto-organisées constituent les formes sociales les plus adaptées à l'émergence, l'accumulation et la circulation des connaissances. Les communautés de savoir deviennent les unités actives de compétence dans les organisations dans nos économies contemporaines (Cohendet, Créplet, Dupouet, 2005). Les travaux s'intéressant à la dynamique des communautés reçoivent un traitement spécifique de la part de l'Ecole du BETA-Strasbourg (Bootz, Burger-Helmchen, Créplet, Cohendet, Dupouët, Guittard, Kern, Munier, Pénin, Schenk). Ils s'intéressent particulièrement à la dynamique des connaissances dans ses rapports à l'autorité. 


\section{7

\subsubsection{Approches des communautés}

L'étude des communautés de pratiques et des communautés épistémiques possède une généalogie aujourd'hui bien identifiée (Tableau 1). L'identification des premières communautés a été réalisée au moyen d'études de cas. On attribue à Orr (1990) la paternité de ces études relatives à l'apprentissage socialement construit par les employés. Plus généralement, la littérature relative aux communautés est présentée comme le produit de quatre lignées de recherches empruntant en grande partie à la sociologie nord-américaine : a) l'apprentissage par la pratique (Brown et Duguid, 1991), b) le caractère situé de la connaissance (Greeno et Moore, 1993), c) l'apprentissage comme construction sociale (Lave, 1988, 1991), d) l'apprentissage comme pratique sociale où se négocient les significations relatives à l'action (Wenger, 1998). Le rapport des communautés aux savoirs est celui du local et de la socialisation. Les communautés sont analysées comme des lieux où se construisent en permanence des modèles locaux, des représentations partagées et des jargons ; en cela, elles favorisent la synergie des variétés individuelles (Amin et Cohendet, 2004). Une communauté se fonde sur des normes auto-développées qui constituent de fait des guides pour l'action. Ne pas se conformer à ces normes conduit à remettre en question la viabilité de l'évolution de la communauté (Munier, 2009).

La plupart des organisations sont constituées de plusieurs communautés. Comment peuvent alors se coordonner organisation fonctionnelle et communautés autonomes?

Tableau 1 - Les deux types de communautés

\begin{tabular}{|l|l|}
\hline \multicolumn{1}{|c|}{ Communautés de pratiques (CPs) } & \multicolumn{1}{|c|}{ Communautés Epistémiques (CE) } \\
\hline $\begin{array}{l}\text { Poursuivent l'objectif d'améliorer l'activité à travers } \\
\text { une réflexion sur les pratiques en construisant et } \\
\text { partageant un répertoire commun de ressources. }\end{array}$ & $\begin{array}{l}\text { De petits groupes de travail composés d'agents } \\
\text { travaillant sur un sous-ensemble mutuellement reconnu } \\
\text { de problèmes liés à un type de connaissances qui au } \\
\text { minimum acceptent une autorité procédurale reconnue } \\
\text { de la même façon par tous et jugée essentielle au } \\
\text { succès de leur activité cognitive (Cowan, David et Foray, } \\
2000) .\end{array}$ \\
$\begin{array}{l}\text { Processus de socialisation qui sont constitués de } \\
\text { savoir-faire tacites, ils sont socialement localisés et } \\
\text { procèdent par répertoires partagés de ressources } \\
\text { communes (routines, artefacts, vocabulaire, styles). }\end{array}$ & $\begin{array}{l}\text { Elles utilisent des Codebook : dictionnaire et ensemble } \\
\text { de règles de grammaire, facilitant la communication et } \\
\text { les synergies. } \\
\text { Elles procèdent par extériorisation pour créer des } \\
\text { connaissances. }\end{array}$ \\
\hline
\end{tabular}

S'intéresser aux communautés implique en fait de se confronter à plusieurs modes d'interaction problématiques. D'une part, les interactions entre structures hiérarchiques posent la question de la diffusion et de la combinaison des connaissances, dans ce cas, les communautés autonomes interviennent de manière limitée. D'autre part, les interactions entre communautés autonomes posent la question du transfert des connaissances avec en arrière fond celle de la pression exercée par la hiérarchie. 


\section{Un état francophone du champ du management des connaissances : la communauté GeCSO}

\subsubsection{L'analyse de l'antinomie hiérarchie-communauté}

Les règles qui président à la hiérarchie et aux communautés sont a priori opposées. La communauté peut recevoir des pressions de son environnement, par exemple d'une hiérarchie au sein de laquelle elle s'inscrit. Mais la communauté est créative au travers de la construction de ses propres normes et plus généralement des interactions entre les membres. La hiérarchie s'intéresse à la communauté afin de développer des activités, d'innover et de contrôler. D'un point de vue synchronique, la relation firme-communauté peut être appréhendée en termes de pression exercée par la première sur la seconde (Munier, 2009). Une communauté est viable dans la mesure où elle respecte des normes sociales définies par ses membres. Ses actions se développent au sein d'un répertoire de normes. En conséquence, la communauté peut se conformer à ces pressions si et seulement si les normes correspondantes aux actions appartiennent au répertoire de normes possibles. Alors qu'une firme tente toujours de tenir une position dirigée par des objectifs, la communauté évolue en fonction de son passé, son action est structurée par des contraintes portant sur ses ressources et l'orientation de ses règles. La firme en tant qu'instance de régulation doit donc en permanence jongler selon une double contrainte : le respect des normes de fonctionnement de la communauté et la possibilité de capter et de diriger cette communauté. On se retrouve donc avec une dualité de structures de gouvernance : d'où l'intérêt développé à propos des figures complémentaires de l'entrepreneur et du manager.

\subsubsection{Entrepreneur et Manager}

L'approfondissement de cette dynamique d'un point de vue managérial est proposé au moyen de la confrontation des rôles de l'entrepreneur et du manager (Tableau 2). D'un point de vue classique et plutôt statique, la communauté peut être envisagée comme un risque de perte de contrôle. Des études approfondies sur des cas particuliers tendent à estomper cette crainte. Le caractère étrange de la communauté de connaissances doit être perçu comme une innovation managériale et non comme un risque organisationnel (Bootz et Kern, 2010). 
Tableau 2 - Rôles de l'Entrepreneur et du Manager

\begin{tabular}{|l|l|}
\hline \multicolumn{1}{|c|}{ Rôle de l'entrepreneur } & \multicolumn{1}{c|}{ Rôle du Manager } \\
\hline $\begin{array}{l}\text { Centré sur la gestion et la diffusion de } \\
\text { nouvelles connaissances, activité plus } \\
\text { tacite, plus difficilement identifiable. }\end{array}$ & Centré sur les liens intérieur/extérieur. \\
$\begin{array}{l}\text { Intention + attention. } \\
\begin{array}{l}\text { Intention : volonté d'imprimer une stratégie } \\
\text { déterminée et d'y engager son entreprise. }\end{array}\end{array}$ & $\begin{array}{l}\text { Assure un environnement interne favorable } \\
\text { à la création des communautés et gère les } \\
\text { connaissances de base par recherche de } \\
\text { codification. }\end{array}$ \\
$\begin{array}{l}\text { Attention : observation et assimilation des } \\
\text { signaux faibles dans l'ensemble des CPs. }\end{array}$ & $\begin{array}{l}\text { Préoccupation de gestion des interfaces pour } \\
\text { conserver le contrôle. }\end{array}$ \\
$\begin{array}{l}\text { L'entrepreneur tente de maintenir la } \\
\text { diversité des connaissances tout en les } \\
\text { faisant dialoguer. }\end{array}$ & $\begin{array}{l}\text { Recours aux objets frontières afin de garantir la } \\
\text { communication. }\end{array}$ \\
\hline
\end{tabular}

Source : d'après Bootz et Kern (2009), Créplet et alii. (2001)

D'un point de vue évolutionniste, plusieurs voies permettant de promouvoir les communautés de savoir sont explorées (Créplet et Dupouët, 2009). Les auteurs recommandent aux hiérarchies de ne procéder à aucune gestion directe des communautés pour, au contraire, créer un contexte favorable à leur développement. Ils préconisent également de ne produire aucune injonction contradictoire de règles et de normes.

\subsection{Fondement épistémologique du management des connaissances}

Il y a enfin des travaux autour d'un thème ayant un caractère plus épistémologique qui sont centrés sur la question de la connaissance en tant que " knowing in action » et qui essayent de dégager les conséquences en matière de management des connaissances de cette perspective.

Le passage de la connaissance comme un objet à la connaissance comme une activité cognitive constitue une rupture épistémologique dont il faut mesurer les conséquences sur le plan théorique en matière de management des connaissances. Ainsi, un certain nombre de travaux abordent la question du management des connaissances du point de vue d'une épistémologie de la pratique. II s'agit de prendre comme point de départ une définition de la connaissance comme liée à une pratique située au sens de "knowing in action » telle qu'elle a été initiée par les travaux de Polanyi dans les années 50. 


\section{Un état francophone du champ du management des connaissances : la communauté GeCSO}

\subsubsection{De la connaissance tacite à la « connaissance en acte »}

Les travaux initiés par Lièvre et Rix-Lièvre $(2008,2010,2012)$ dans le champ des «Organization Studies » proposent de donner un contenu plus théorique et plus précis à la notion de connaissance tacite. Ils préfèrent parler de « connaissance en acte » au sens de Piaget et considèrent que le « tacite » est une caractéristique d'une " connaissance en acte », et encore plus précisément que le bon vocabulaire pour parler de cet aspect non explicite des « connaissances en acte » est « implicite ». Nous sommes ici sur le terrain des connaissances strictement individuelles. Ils proposent de réserver l'usage de la dimension tacite d'une connaissance pour rendre compte d'une caractéristique d'une connaissance collective comme on le fait dans le domaine juridique lorsque l'on désigne quelque chose de connu par différentes parties mais non explicité à un moment donné. L'objet de ces travaux est de reprendre le fil de l'intuition de Polanyi et de montrer en quoi la mobilisation des travaux de Piaget des années 70 autour du thème "réussir et comprendre » ainsi que des travaux d'ordre méthodologique développés par Pierre Vermersch dans une perspective psycho-phénoménologique dans les années 90 peuvent participer d'une manière féconde à la clarification de ces notions : connaissance tacite et connaissance explicite qui sont des notions fondatrices en management des connaissances. L'idée de base est de considérer les pratiques et donc les connaissances liées à ces pratiques comme relevant d'un niveau de conscience pré-réfléchie et donc non consciente, opérant une distinction fondamentale sur le plan épistémologique entre l'agir et la conscience de l'agir. Quand je lis, je lis. Je n'ai pas besoin d'avoir conscience que je lis pour lire. Ce qui ne veut pas dire que je ne peux revenir consciemment sur ma manière de lire pour progresser. L'acteur n'est pas en mesure spontanément de rendre compte de ces connaissances car il rationnalise, il met en cohérence son vécu en fonction de son interlocuteur et de son identité. II faut alors construire un dispositif spécifique pour accéder à ces « connaissances en acte » : entretien d'explicitation (Vermersch, 1996) entretien d'auto-confrontation (Theureau, 1992), entretien en re-situ subjectif (Lièvre-Rix, Lièvre, 2008). On ne peut accéder à ce type de connaissance par un simple entretien sociologique classique conduit après l'action. Ces auteurs proposent de développer les conséquences pour le management des connaissances de ce type de position théorique, par exemple sur la question de la codification.

\subsubsection{Connaissance fondamentale, routinière, spécifique}

Les travaux de Hakim Hachour et Safia Abouad (2008) en sciences de l'information et de la communication, issus du laboratoire Paragraphe de l'Université Paris 6 qui s'inscrivent dans une tradition socio phénoménologique initiée par l'œuvre de Schultz $(1953,1976)$ qui permettent de distinguer trois types de connaissance : connaissance fondamentale, connaissance routinière, connaissance spécifique. Les travaux de Schutz $(1953,1976)$ et de Schutz et Luckman (1973) définissent trois types de connaissance et s'intéressent au style cognitif qui permet d'accomplir un but. Ce processus se structure en étapes dont les articulations doivent être 


\section{7

étudiées en détail. Cette approche (" course-of-action patterns ») sera reprise et développée par Theureau (2006). Les connaissances tacites ou fondamentales sont les connaissances prises comme allant de soi. Elles procèdent d'un arrangement de processus expérimentés de manière purement subjective. Les connaissances spécifiques sont détachées de la situation, elles sont objectives. Les connaissances routinières sont liées aux finalités pratiques de l'activité, elles se réfèrent aux possibilités de réalisation que contient l'environnement quotidien (significations en action) selon Schutz (1953, 1976).

Ces distinctions essentielles facilitent l'éclairage de travaux touchant aux sciences de gestion. Elles facilitent également l'approfondissement d'une possible « gestion des connaissances » par l'association de travaux développés en sociologie phénoménologique et en psychologie phénoménologique. A partir de ces principes et d'une étude sur l'organisation naturelle d'un système de connaissances socialisées, ils se proposent de concevoir un dispositif de gestion des connaissances comme une réorganisation " centrée mémoire " au sens de Le Moigne qui implique trois niveaux de problèmes fondamentaux sur le plan de la modélisation : le registre syntaxique qui est celui de la technologisation, le registre sémantique qui est celui de la référentialisation, et le registre pragmatique qui est celui de l'implication ; eux-mêmes soumis à un méta-niveau de problèmes primordial : l'éthique.

\subsubsection{Deux perspectives d'étude des processus cognitifs}

Les travaux de Brassac (2008) complèteront le propos en distinguant deux perspectives d'étude des processus cognitifs : la perspective « internaliste » qui conçoit la cognition comme un système de traitement d'informations opérant sur des entités intracrâniennes et des états mentaux correspondant à des états du monde que le sujet tente de comprendre, mémoriser, dire et manipuler ; la perspective " externaliste " qui relie ensemble cerveau, corps et monde. La cognition est alors une activité qui rend "présente » les propriétés du monde au lieu de les représenter. Nous pouvons maintenant aborder les trois autres programmes de recherche plus spécifiques à des opérations de management des connaissances dans les organisations.

\section{Des programmes liés à des opérations spécifiques de management des connaissances}

\subsection{La génération des connaissances}

Un ensemble de travaux s'intéressent à la génération des connaissances dans les organisations. Certains se centrent sur la génération des connaissances au sein d'activité de conception et tentent de répondre à la question : comment permettre l'expansion des connaissances dans une démarche de projet ? 


\section{Un état francophone du champ du management des connaissances : la communauté GeCSO}

D'autres envisagent cette question sur l'ensemble du cycle de vie d'un produit au sein d'une organisation.

\subsubsection{La génération des connaissances au sein de la conception}

II s'agit en fait de concilier différentes temporalités au sein de l'organisation : celle des activités ou des projets et celle de la capitalisation des connaissances. Un exemple permet d'en apprécier la portée. II s'agit du modèle de conception à base de réseau de contradictions développé dans le cadre du programme Myriade (conception de microsatellites) du CNES, étudié par Belleval et alii. (2010). Ce programme engage ses acteurs dans une évolution de leurs théories de l'action au sens d'Argyris et Schön (1978). II s'agit de passer d'une représentation de type "Mission Success First » privilégiant la fiabilité et la robustesse des systèmes à une autre représentation de type « Smaller, Faster, Better, Cheaper » inspirée par la politique menée par Dan Goldin, administrateur de la NASA au début des années 1990. Cette évolution a toute son importance dans le management du processus de conception. Dans ce cas, le problème de conception n'est pas encore bien défini et des contradictions peuvent apparaître en cours de déroulement. II s'agit d'assumer cet état de fait tout en dépassant le modèle " problem solving » initié par Simon (1972). Différentes théories de la conception sont mobilisées à cet effet, elles permettent de mettre en évidence l'interdépendance entre trois espaces (Pimmler et Epinger, 1994) : celui du produit (quoi faire?), celui du projet de conception (comment faire?), celui des acteurs (qui fait ?). L'interaction entre les activités de résolution des problèmes techniques et l'organisation du processus de conception a un impact sur le nombre d'itérations dans le processus de conception et sur la performance du projet (coût, délais, qualité). En assumant les perturbations qui peuvent affecter les relations entre ces différentes "dimensions », on adopte du même coup une nouvelle définition de la conception innovante. Son processus est non structuré car son objectif se construit durant le déroulement des activités. Le problème à résoudre est contradictoire : un ensemble de contradictions techniques, organisationnelles et cognitives doit être levé (Belleval et Lerch, 2010). L'ingénierie système se prête à une telle démarche avec notamment le modèle classique de développement en V de Forsberg et Mooz (1991). Ce modèle permet la co-évolution entre la structuration du problème et la solution. L'analyse des besoins conduit à préciser les spécifications techniques qui peuvent se révéler contradictoires. C'est lors de la conception de l'architecture qu'il va falloir gérer les contradictions. Pour cela on va soit exploiter une base de connaissances connues, soit abandonner le projet car irréalisable, soit encore exploiter les contradictions afin de créer de nouvelles combinaisons (Altshuler, 1984), en procédant à une expansion de l'espace des solutions (Le Masson et alii., 2006).

Dans chacun des cas, on prend en charge les interactions entre les caractéristiques techniques du produit, l'organisation du projet de conception etl'organisation même de l'entreprise, notamment de ses acteurs. Ces trois dimensions se déploient 


\section{7

selon plusieurs cycles en $\mathrm{V}$ dans la structuration de classes de conception. Le problème de conception est donc un espace ouvert qui offre des opportunités de résolution de problèmes/contradictions par expansion des dimensions cognitive, technique et organisationnelle. Cette étude permet de mettre en évidence qu'un blocage au niveau d'une seule contradiction peut aboutir à l'échec relatif du projet (une innovation incrémentale à la place d'une innovation radicale). C'est donc la maîtrise des interactions entre les trois dimensions qui est stratégique dans l'orientation du projet de conception. Ce résultat de recherche nous conduit à une certaine généralisation dans la tentative de génération des connaissances sur le cycle de vie d'un produit.

\subsubsection{La génération des connaissances sur le cycle de vie d'un produit}

C'est la démarche développée par l'Ecole Bordelaise (Brunel, Girard, Moradi, Vallespir, Zolghadri, 2008, 2010) qui vise l'intégration systémique et tente de modéliser un cycle dit "d'Ingénition ». Ces chercheurs s'inscrivent dans la démarche d'ingénierie et partent du constat que différentes théories complémentaires s'ignorent en fait : celles de la conception, de la connaissance et de l'apprentissage. A partir de plusieurs interventions en milieu industriel, ils établissent qu'il est faux de penser que des spécialistes "bien formés » savent gérer leurs apprentissages et connaissances. II s'agit de sauvegarder la connaissance endogène en prenant en charge l'ensemble de la chaîne de valeur développée par une organisation particulière. Pour cela, on construit un filtre « objet - sémantique - social et structurel » qui permet de développer progressivement une génération de produits avec les données, les informations puis les connaissances. Finalement il s'agit de mettre en commun des connaissances, des compétences et des acteurs tout au long de la chaîne de valeur. L'enjeu est de s'assurer que l'on dispose des bases de connaissances, ou que l'on pourra les mobiliser à l'extérieur, au moment de la conception et du développement d'un produit. Le processus d'intégration des connaissances se déploie en cinq phases : a) modélisation du contexte, b) conception de l'utilisation du produit par le client, b) génération de la connaissance au cours du cycle de vie du produit étendu, d) échelle comparative des moyens internes et externes, e) chaîne de valeur séquences de valeur - génération de connaissances. Le développement de ce processus doit finalement faciliter l'intégration des ressources et des capacités cognitives de la firme.

\subsection{Le transfert des connaissances}

Un programme sur le transfert des connaissances qui prend différentes orientations : a) le processus même de transfert de connaissance sous l'angle de la psychologie social des processus cognitifs (Brassac, 2008), b) le transfert par le développement des compétences (Coulet, 2011) et enfin c) le transfert des connaissances au moyen de dispositif de formation (Wannemacher, 2010 ; Gadille, Machado, 2011). 


\section{Un état francophone du champ du management des connaissances : la communauté GeCSO}

\subsubsection{La transmission de connaissances entre Connaissant et Apprenant}

La recherche de conditions spécifiques pour assurer un transfert considéré comme critique au sein d'une organisation fournit l'occasion d'approfondir les modalités du processus de transfert proprement dit. Brassac $(2000,2008)$ développe une méthodologie d'aide au transfert à partir d'une approche psychologie sociale des processus cognitifs. Les situations les plus typiques sont celles du départ d'un technicien ou d'un ingénieur. L'acquisition de connaissances est un processus dont deux acteurs au moins (A)pprenant et (C)onnaissant sont co-responsables. Ce processus se déploie dans un ensemble d'interactions sociales, constituées des échanges discursifs, des productions gestuelles, et par des manipulations de machines. Pour les acteurs, il s'agit de maintenir ces interactions. Dans ces conditions, il n'existe pas à proprement dit de transfert de connaissances. II se développe plutôt une construction conjointe de significations ayant vocation à être utilisées et appropriées par $\mathrm{A}$ dans l'après-coup de l'acquisition. Afin de faciliter cette appropriation, Brassac développe la méthodologie suivante : a) le recueil du discours n'est pas suffisant, b) il ne faut pas déconnecter le discours de $C$ et l'appréhension de ce discours par A, c) il ne faut pas abstraire l'expression de l'expertise de son lieu concret d'effectuation, d) il ne faut pas empêcher les deux acteurs $C$ et $A$ de représenter graphiquement les éléments de travail. Ainsi on donne l'occasion à $\mathrm{C}$ et $\mathrm{A}$ d'avoir un rapport direct : rapport aux savoirs, aux hésitations, aux oublis, dépendance aux documents et aux limites. On préserve la possibilité d'interroger, de soulever des incohérences, mettre à jour des conflits et proposer des perspectives nouvelles. Pour cela il est nécessaire de travailler à trois (C, A, le chercheur-intervenant ou un interviewer), on utilise des mises en situation en contexte concret de travail, des films, des entretiens et des entretiens d'auto-confrontation face aux films. Les perspectives de transfert sont donc liées au fait que la connaissance est distribuée entre $C$ et les artefacts (documents, équipements... ${ }^{47}$. Une bonne stratégie consistera alors à privilégier les allers-retours entre les différentes modalités de " recueil » des connaissances (diversité, redondance). Ce qui permet de relever les décalages entre énoncés performatifs et expériences et de produire des recommandations et reformulations performatives.

En résumé, deux préconisations pratiques sont formulées. II faut « retenir » : en organisant la documentation, retenir l'expertise, faire face aux aléas, organiser les ressources après analyse des corpus. Ce qui relève d'une psychologie de l'interaction et du travail. II faut " capitaliser » : en sauvegardant les protocoles d'usage, anticiper les transmissions futures, concevoir un dispositif de sauvegarde de l'activité de A.

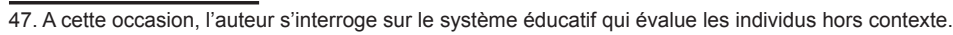




\section{7

\subsubsection{Transfert par développement des compétences}

Coulet (2011a, 2011b) développe un modèle de la compétence qui permet d'envisager des modèles de tutelle. Partant d'un constat détaillé d'insuffisance des approches concurrentes en matière de compétence, et en prenant appui sur Rogalski (2004) l'auteur développe la notion de « double régulation de l'activité par la situation et le sujet » afin de rendre justice à la richesse des activités d'interprétation et d'analyse des situations.

Le modèle est centré sur les différentes dimensions du "cours d'activité ": l'activité fait l'objet d'une organisation hiérarchisée, elle peut faire l'objet d'une pluralité de modes de réalisation, enfin, elle possède deux faces : elle permet d'obtenir un résultat tout en participant à la construction du sujet (association des " activités productives " et " activités constructives " selon Samurcay et Rabardel, 2004). Ce modèle est situé selon des schèmes définis comme des entités dynamiques fonctionnelles (Tableau 3) ou « organisation invariante de la conduite pour une classe de situations données » (Vergnaud, 1990).

Tableau 3 - Les quatre éléments d'un schème

\begin{tabular}{|l|l|}
\hline \multicolumn{1}{|c|}{ Elément } & \multicolumn{1}{c|}{ Composition } \\
\hline Invariants opératoires & $\begin{array}{l}\text { ce que le sujet tient pour vrai (" théorèmes en-acte») et ce que le } \\
\text { sujet tient pour pertinent (« concepts-en-acte »). }\end{array}$ \\
\hline Inférences & $\begin{array}{l}\text { prises d'informations, calculs et contrôles permettant l'ajustement, } \\
\text { ici et maintenant, du schème aux variables de situation. }\end{array}$ \\
\hline Règles d'action & $\begin{array}{l}\text { composantes effectrices, engendrent des suites d'actions } \\
\text { conduisant au résultat recherché. }\end{array}$ \\
\hline Anticipations des résultats & liées au but visé par la mobilisation du schème. \\
\hline
\end{tabular}

Source : Coulet (2011a)

Dans ce cadre, la compétence est définie comme « une organisation dynamique de l'activité, mobilisée et régulée par un sujet pour faire face à une tâche donnée, dans une situation déterminée ". Il est possible d'envisager les dynamiques d'intégration connaissances - compétences dans l'organisation de l'activité d'un sujet. On rend compte des relations entre les différents composants (Invariants opératoires, Inférences, Règles d'action, Anticipations) permettant d'assurer la réalisation d'une tâche dans une situation déterminée. Ces interactions marquent la mobilisation sur une tâche et dans une situation déterminée, d'un schème (via un ou plusieurs artefacts) dont les composantes assurent la réalisation, ajustée aux caractéristiques spécifiques de la situation et de la tâche, ce qu'on peut considérer comme une activité productive. Le résultat ainsi obtenu fournit généralement, de façon directe ou indirecte, des informations en retour (feedback) qui permettent au sujet de mettre en place différents types de régulations (correspondant à l'activité constructive). Dans un tel schéma d'actions, un 


\section{Un état francophone du champ du management des connaissances : la communauté GeCSO}

tuteur va pouvoir situer précisément la dimension visée par l'apprentissage et le transfert de connaissances. On se situe ainsi très loin de la conception « expert à imiter » puisqu'on va privilégier des processus de mobilisation de schèmes et de construction par étapes.

\subsubsection{Transfert de connaissances au moyen des dispositifs de formation}

Wannenmacher (2010) présente une large synthèse de ces dispositifs : tutorat, doublure et compagnonnage. La fonction " tutorale " est un processus de socialisation professionnelle ainsi qu'un processus de transmission des pratiques professionnelles. Le tuteur guide le nouvel arrivant et l'aide à surmonter ses difficultés dans son apprentissage (Boru et Leborgne, 1992). La fonction « doublure » permet de faciliter la socialisation du nouvel arrivant. Celui-ci est mis en double commande avec un salarié expérimenté pendant toute la période de prise de poste (Meignant, 2001). Enfin, la fonction « compagnonnage » permet la diffusion de tours de main, de savoir-faire ou de compétences techniques au sein des collectifs de travail correspondant à des métiers spécifiques.

Ces trois fonctions visent à instaurer des conditions favorables aux interactions. Les savoir-faire ne peuvent se transmettre sans l'existence d'actions simples telles que l'observation, l'imprégnation, l'imitation, l'expérience concrète, le conseil des anciens, la démonstration, les essais-erreurs et exercices. Encore faut-il que les conditions soient établies, lorsqu'elles le sont, l'apprentissage peut avoir lieu. Dans une perspective voisine, Gadille et Machado (2011) s'intéressent au processus de concrétisation, non pas des objets techniques au sens de Simondon (1989), mais à celui des systèmes de transfert des connaissances. Ils étudient les décalages entre méthodes de management des connaissances et pratiques individuelles des salariés. Ils proposent une méthode de transmission des savoirs rares, inspirée de la recherche intervention en sociologie clinique, dite " méthode semi-directive » dont le but est de constituer des "Guides des Savoirs " afin de faciliter l'apprentissage et la transmission de connaissances par des experts aux apprenants. Le transfert est envisagé dans ses dimensions sociales et organisationnelles, il est considéré comme un moyen pour faire évoluer les relations au sein de l'organisation.

\subsection{La capitalisation des connaissances}

Les travaux de recherche centrés sur la capitalisation des connaissances ont deux axes : le programme autour des retours d'expérience (REX ou RETEX) (Gautier, 2010 ; Godé et Barbaroux, 2011 ; Rachedi, Boujut, Poussier, 2010) et celui de la captation des gestes techniques à partir de dispositif vidéo (Le Bellu, Lahlou, Le Blanc, 2009). 


\section{7 \\ management \\ Qr avenir}

\subsubsection{Le retour d'expérience}

Les travaux de Gautier (2010) s'intéressent aux pratiques du retour d'expérience et à leur formalisation dans les organisations. Elle vise à déterminer un savoirfaire scientifique et managérial par la description des modalités de mise en œuvre du retour d'expérience dans une perspective d'apprentissage organisationnel. La réflexion accorde un rôle essentiel au formalisme des pratiques du retour d'expérience pour la production d'une connaissance sur l'organisant. C'est le retour d'expérience des sapeurs-pompiers sur des situations critiques de feux de forêt qui constitue le terrain de la recherche. D'autres travaux sur le terrain comme celui de l'armée de l'air proposent d'interroger le retour d'expérience dans sa capacité à produire du sens dans les organisations, à gérer des paradoxes dans l'organisation, à fabriquer des compétences (Barbaroux, Godé, 2010 ; Godé, 2012). Dans un contexte d'ingénierie travaillant sur des objets métiers, la gestion des connaissances consiste à permettre à l'acteur de donner du sens aux informations liées à ses objets métiers et de naviguer contextuellement entre les différents objets qu'il manipule dans son activité. Ainsi l'outil TEEXMA (Rachedi et alii., 2010) est utilisé en tant que dispositif support dans le cadre du Retour d'Expérience (ou REX). L'outil permet de déclarer factuellement une situation, attachée à un objet métier, qui est analysée collectivement, et fait l'objet de la création d'un savoir court (un retour d'expérience), qui peut être documenté, et qui doit être facilement appropriable par les acteurs concernés, en vue d'une amélioration continue de l'activité.

\subsubsection{Capter les gestes techniques}

Une autre illustration de capitalisation des connaissances techniques est fournie par les outils vidéo. Elle est particulièrement développée en secteur sensible (énergie, nucléaire). II s'agit de développer des moyens de capture du savoir-faire incorporé dans les gestes métiers dits « rares » (exemple d’EDF). Une réflexion est très poussée dans ce sens en matière de diversité et de qualité des prises de vues, ainsi qu'à propos des modes d'explicitation par les acteurs en cours d'enregistrement (Le Bellu, Lahlou et Le Blanc, 2009). L'approche développée par EDF est fondée sur une vue subjective de l'activité de l'opérateur et sur un protocole permettant de recueillir les éléments cognitifs subjectifs de l'exécution du geste en contexte. Deux caméras sont utilisées, l'une en plan large, l'autre portée par l'opérateur au plus proche de ses gestes. Le choix de prise de vue « à la première personne " permet d'accentuer le processus d'identification à l'opérateur de la part de l'observateur-spectateur. II fournit également des possibilités d'amélioration de la qualité de l'explicitation par l'opérateur. Les questions qui sont posées par ce dispositif sont les suivantes : l'opérateur tendt-il à appliquer des routines de façon inconsciente (en proportion d'ailleurs de son expertise) ? Comment les lui faire expliciter en cours d'action ? Quelles en sont les limites ? Se pose alors la question du type de caméra, de l'angle de vue et du type d'enregistrement. Au moyen d'une préparation mentale, il est 


\section{Un état francophone du champ du management des connaissances : la communauté GeCSO}

demandé à l'opérateur-formateur de penser son geste, de se mettre en position réflexive sur son geste (annonce des buts et des résultats séquentiellement) afin qu'il en fournisse la meilleure description possible et ce dans un format qui permette une modélisation cognitive du geste puis une structuration des supports pédagogiques.

\section{Conclusion}

Il est possible de dégager trois types de résultat de cette première structuration du champ du management des connaissances de la communauté GecSO. Le premier tient au caractère multidisciplinaire du champ mais qui nécessite de passer au-delà de cette multidisciplinarité, c'est-à-dire de la multiplication des angles de vue disciplinaires sur le même objet. Les différents programmes de recherche dont nous avons rendu compte dans cet article font apparaître des nombreuses articulations disciplinaires. Prenons pour exemple, le programme de l'économie de la connaissance aux communautés de savoir. Ce programme démarre dans le champ de l'économie pour aller dans le champ de la sociologie pour enfin interpeller le champ de la gestion. Tout se passe comme si on ne pouvait enfermer le champ du management des connaissances dans une seule discipline que par nature les programmes de recherche qui s'inscrivent dans ce champ ont une forte composante d'interdisciplinarité voire de même de transdisciplinarité. Cette situation d'indisciplinarité, si inconfortable et si riche, est liée à la nature même de l'objet de recherche : le couple connaissance-organisation ainsi que son pilotage. Deuxième résultat, il y a des convergences dans les différents programmes de recherche comme sur le fait de distinguer d'une manière très nette l'information et la connaissance, de considérer la connaissance comme une activité cognitive plutôt qu'un objet, mais il y a aussi des liens entre ces différents programmes qui permettent de faire émerger une architecture d'ensemble ce que nous avons essayé de faire en croisant ces six programmes de recherche sous la forme d'une matrice. Enfin dernier résultat, il y a une évolution du domaine du management des connaissances qui au départ avait une position plutôt défensive en développant des approches à même de conserver le capital critique des connaissances dans les organisations à une position plus offensive où le développement des connaissances devient le support à l'innovation. Nous mesurons au terme de cet article le chemin à parcourir pour structurer le champ du management des connaissances. Nous proposons ici une itération dans un processus de structuration du champ du management des connaissances au sein de la communauté GecSO.

\section{Bibliographie}

AMIN A., COHENDET P. (2004), Architecture of Knowledge, Oxford University Press, Oxford.

ARGYRIS C., SCHÖN D. (1978), Organizational Learning, Addison-Wesley, Reading, MA. 


\section{7 \\ R E V U E \\ management \\ \& avenir}

AUBERTIN G. (2007), « Cartographier les connaissances critiques: une démarche stratégique pour l'entreprise ». In Management des connaissances en entreprise. Lavoisier. Paris, Hermès Science, p. 125-144.

BELLEVAL C., DENIAUD I., LERCH C. (2010), « Modèle de conception à base de réseau de contradictions. Le cas de la conception des microsatellites au CNES », Actes du $3^{\text {ème }}$ Colloque GeCSO, Université de Strasbourg.

BOOTZ J.-P., KERN F. (2010) "Les communautés de connaissance : leviers de changement organisationnel pour le manager et l'entrepreneur ", Actes du $3^{\text {ème }}$ Colloque GeCSO, Université de Strasbourg.

BOOTZ J.-P. et KERN F. (2009) Les communautés en pratique : leviers de changements pour l'entrepreneur et le manager, Editions Hermès \& Lavoisier.

BORU J.-J. et LEBORGNE C. (1992), « Introduire et développer la fonction tutorale dans les entreprises ", Actualité de la Formation Permanente, n¹19, p. 24.

BOURDIEU P. (1994), Raisons pratiques, Editions du Seuil, Paris.

BRASSAC C. (2008) « L'acquisition de savoirs comme activité située et distribuée », Actes du $1^{\text {er }}$ Colloque GeCSO, ESC Troyes.

BRASSAC C. (2000), "Intercompréhension et Communiaction® », in A.-C. Berthoud, L. Mondada (éds), Modèles du discours en confrontation, Berne, Peter Lang, p. 219-228.

BRASSAC C. (2004), "Action située et distribuée et analyse du discours : quelques interrogations », Cahiers de Linguistique Française, 26, p. 251-268.

BROWN J.-S., DUGUID P. (1991), Organizational learning and communities-of-practice: Toward a unified view of working, learning and innovation », Organization Science, Vol. 2, $\mathrm{n}^{\circ} 1$, p. $40-57$

BROWN J.-S., DUGUID P. (2001), « Knowledge and Organization: A Social-Practice Perspective », Organization Science, Vol. 12, n², p.198-213.

BRUNEL S. (2008), Etude des activités collaboratives de conception en tant que situation d'apprentisage : application à l'ingénierie des produits et à l'ingénierie didactique. Thèse $N^{\circ} 3680$, Bordeaux 1 University, France.

BRUNEL S., ZOLGHADRI M., GIRARD P. (2008), Ingénition : une méthode stratégique pour la génération de la connaissance, Actes du 1 er Colloque GeCSO, ESC Troyes.

CHOULIER D. (2008), Comprendre l'activité de conception, Collection "Chantiers ", Université de Technologie de Belfort-Montbéliard.

COULET J.-C. (2011), « La notion de compétence : un modèle pour décrire, évaluer et développer les compétences ", Le travail humain, Vol. 74, n¹, p. 1-30.

COULETJ.-C. (2011), «L'expert-compétent: une perspective piagétienne », communication au $4{ }^{\text {ème }}$ Colloque GeCSO, Groupe ESC Clermont-Ferrand, 19 et 20 mai.

COWAN R., DAVID P., FORAY D. (2000), "The explicit economics of knowledge codification", Industrial and Corporate Change, 9, 2, p.211-253.

CRÉPLET F., DUPOUET O., KERN F. et MUNIER F. (2001), " Dualité cognitive et organisationnelle de l'entreprise : le rôle différencié du manager et de l'entrepreneur ", Revue d'Économie Industrielle, $\mathrm{n}^{\circ}$ 95, p. 9-22.

CRÉPLET F., DUPOUET O. (2009), « Communautés d'apprentissage et pratiques prospectives : de l'aide à la décision au management des connaissances » in Bootz J.-P. et Kern F., Les communautés en pratique : leviers de changements pour l'entrepreneur et le manager, Editions Hermès - Lavoisier.

DIENG-KUNTZ R. (2007), Capitalisation des connaissances via un web sémantique d'entreprise. Management des connaissances en entreprise, Lavoisier \& Hermès Science, Paris, 255-272.

ERMINE J.-L. (1996), Les systèmes de connaissances, Editions Hermès, Paris.

ERMINE J.-L. (2003), Gestion et Ingénierie des connaissances, modèles et méthodes, Edition Hermès, Paris.

ERMINE J.-L. (2007), « Introduction au Knowledge Management » in Management des 


\section{Un état francophone du champ du management des connaissances : la communauté GeCSO}

connaissances en entreprise, Lavoisier \& Hermès Science, Paris, p. 23-45.

ERMINE J.-L. (2008), Management et ingénierie des connaissances, modèles et méthodes, Hermès Science publications, $376 \mathrm{p}$.

FORSBERG K., MOOZ H. (1991), "The Relationship of System Engineering to the Project Cycle", INCOSE, p. 57-65.

GREENO J.-G. et MOORE J.-L. (1993), « Situativity and symbols : Response to Vera and Simon », Cognitive Science, n¹7, pp. 49-59.

GRUNDSTEIN M. (2002), Gameth : un cadre directeur pour repérer les connaissances cruciales pour l'entreprise, Lamsade Université Paris-Dauphine : 18 pages.

HACHOUR H., ABOUAD S. (2008), Une contribution de la sémiotique des systèmes d'action à la gestion des connaissances : trois niveaux de problèmes fondamentaux, $1^{\text {er }}$ Colloque GeCSO, Groupe ESC Troyes, France.

JACQUIER-ROUX V., MONTANA N.-C. et PARAPONARIS C. (2011), Réseaux sociaux d'innovation et management de la diversité, 4eme Colloque GeCSO, Groupe ESC Clermont-Ferrand, France.

KERVERN G.-Y. (1995), Éléments fondamentaux des cindyniques, Economica, Paris.

LAVE J. (1988), Cognition in Practice. Cambridge : Cambridge University Press.

LE BELLU S., LAHLOU S. et LE BLANC B. (2009), " Comment capter le savoir incorporé dans un geste technique à l'aide d'outils numériques ? ", Actes du $3^{\text {ème }}$ Colloque GeCSO Gestion des Connaissances, Bordeaux.

LE BOTERF G. (2008), Travailler efficacement en réseau une compétence collective, Eyrolles Editions d'organisation.

LEMASSON P., WEIL B. et HATCHUELA. (2006), Les processus d'innovation : conception innovante et croissance des entreprises, Hermès.

LE MOIGNE J.-L. (1994), La théorie du système général : théorie de la modélisation, PUF, (4e éd.), Paris.

LIĖVRE P. et BONNET E. (2010), Retour sur la mise en œuvre de la méthode MASK à la conduite du blé biologique : la question du mode d'investigation des connaissances tacites, Actes du $3^{\text {ème }}$ Colloque GeCSO Gestion des Connaissances, Université de Strasbourg.

LIĖVRE P. et RIX-LIĖVRE G. (2010), " La dimension " tacite » des connaissances expérientielles : une mise en perspective théorique et méthodologique ", Actes du $3^{\text {ème }}$ Colloque GeCSO Gestion des Connaissances, Université de Strasbourg.

LIĖVRE P., RIX-LIĖVRE G. (2012), « La dimension tacite des connaissances expérientielles individuelles : une mise en perspective théorique et méthodologique ", Management International, Vol 16, Numéro spécial, Gestion des connaissances dans la société et les organisations, p. 21-29.

MEIGNANT A. (2001), « Former sans la formation ? », Personnel, n421, juillet, p. 29.

MENAOUER B., BAGHDAD A. (2009), « Vers une cartographie des connaissances guidée par fouille de données "Actes du $3^{\text {ème }}$ Colloque GeCSO Gestion des Connaissances, Bordeaux.

MUNIER F. (2009) « Viabilité des communautés de pratique pilotée : un modèle dynamique », Actes du $2^{\text {ème }}$ Colloque GeCSO Gestion des Connaissances, BEM, Bordeaux.

ORR J. (1990) " Sharing knowledge, celebrating identity: war stories and community memory in a service culture ", Collective remembering, D.-S. Middleton and D. Edwards, eds., Sage, Beverly Hills, CA, pp. 169-189.

PARAPONARIS C. (2011), " L'état de la recherche francophone en matière de gestion des connaissances ", communication au $4^{\text {ème }}$ Colloque Francophone "Gestion des connaissances, Société et Organisations », ESC Clermont-Ferrand, 19 et 20 mai.

PARAPONARIS C., ERMINE J.-L., GUITTARD C. et LIĖVRE P. (2012), « Knowledge Management in a French Research Community - A Case Study of GeCSO Congress ", Journal of Information and Knowledge Management Systems, Volume 42, $\mathrm{n}^{\circ} 3$, November. 


\section{7

PIAGET J. (1974a), La prise de conscience, PUF, Paris.

PIAGET J. (1974b), Réussir et comprendre, PUF, Paris.

PIMMLER T.-U. et EPPINGER S.-D. (1994), "Integration Analysis of Product Decompositions", Proceedigs of the ASME Design Theory and Methodology Conference, vol. 68, p. 343-351.

POLANYI M. (1962), Personal knowledge: towards a post-critical philosophy. New York: Harper Torchbooks.

RACHEDI S., BOUJUT J.-F., POUSSIER G. (2010), « Etude de cas d'une offre industrielle de management des connaissances techniques ", Actes du $3^{\text {ème }}$ Colloque GeCSO Gestion des Connaissances, Université de Strasbourg.

RIX G., LIĖVRE P. (2008), " Towards a codification of practical knowledge », Knowledge Management Research \& Practice, 6, 225-232.

ROGALSKI J. (2004), « La didactique professionnelle : une alternative aux approches de " cognition située » et « cognitiviste » en psychologie des acquisitions », @ctivites, 1, 2, 103-120. http://www.activites.org/v1n2/Rogalski.pdf

SAMURCAY R. \& RABARDEL P. (2004), " Modèles pour l'analyse de l'activité et des compétences, propositions », in R. Samurcay \& P. Pastre (Eds.) Recherches en didactique professionnelle, p. 163-180, Toulouse : Octarès.

SAULAIS P. et ERMINE J.-L. (2011) « Créativité et gestion des connaissances ». In GeCSO 2011, Gestion des Connaissances pour la Société et les Organisations, ClermontFerrand, France.

SCHENK E., GUITTARD C. et COHENDET P. (2010), "TIC et communautés : le forum comme espace de socialisation ", Actes du $3^{\text {ème }}$ Colloque GeCSO Gestion des Connaissances, Université de Strasbourg.

SCHÖN D. (1983), The reflective practitioner. How professional think in action, Basic Books, New York.

SCHÖN D.-A. (1996), The reflective practitioner, How Professionals Think in Action, Ashgate Publishing Limited.

SCHUTZ A. (1953), "Common-sense and Scientific Interpretation of Human Action". In The Problem of Social Reality, Collected Papers I, La Hayes : Martinus Nijhoff, 1962, pp.3-47.

SCHUTZ A. (1976), The Phenomenology of the Social World [1932]. Londres : HEB.

SCHUTZ A. \& LUCKMANN T. (1973, 1989), The Structures of the Life-World (Volume I et II). IL, Evanston : Northwestern University Press.

SIMON H. et NEWELL A. (1972), Human problem solving, Prentice Hall, Englewood Cliffs, N.J.

SIMON H.-A. (1978), "Rationality as Process and Product of Thought », American Economic Review, Vol. 68, n², pp. 1-16.

SIMONDON G. (1989), Du mode d'existence des objets techniques, Editions Aubier, Paris, première édition 1958.

SOULIGNAC V., ERMINE J.-L., PARIS J.-L., DEVISE O. et CHANET J.-P. (2010), "Gestion informatisée des connaissances pour une agriculture durable », Actes du 3ème Colloque GeCSO Gestion des Connaissances, Université de Strasbourg.

TAN PHAM T.-D., ANTOINE A. (2012), « La génération des connaissances au secours du management de projet, le cas du système d'information d'un établissement de santé ", Management International, Vol 16, Numéro spécial, Gestion des connaissances dans la société et les organisations, p.75-88.

THEUREAU J. (2006), Le cours d'action: méthode développée, Octares, Toulouse.

VERGNAUD G. (1990), "La théorie des champs conceptuels », Recherche en didactique des mathématiques, 10, 2/3, 133-170.

VERMERSCH P. (2003), L'entretien d'explicitation, ESF.

WANNENMACHER D. (2010), " Le partage de connaissances tacites : l'importance 


\section{Un état francophone du champ du management des connaissances : la communauté GeCSO}

de l'interaction in situ ", Actes du $3^{\text {ème }}$ Colloque GeCSO Gestion des Connaissances, Université de Strasbourg.

WENGER E. (1998) Communities of practice: learning, meaning and identity. New York : Cambridge University Press. 